\title{
PANEL DISCUSSION ON THE QUANTUM STATISTICAL AND THERMAL TREATMENTS OF PROCESSES IN MOLECULE-SIZED PAHS
}

\author{
SYDNEY LEACH \\ Département Atomes et Molécules en Astrophysique \\ Meudon, France \\ and \\ Laboratoire de Photophysique Moléculaire du C. N. R.S. \\ Orsay, France
}

This discussion followed the two talks, one by John Barker on "Grains or Molecules, Thermal or Non-Thermal," the second, by Louis d'Hendecourt on "The Particle/Thermal Approach to the Physics of Emission from Interstellar PAHs." The presentations discussed the factors involved in the process of absorption of an ultraviolet photon by a PAH molecule or ion, followed by infrared and/or visible emission. Model calculations were presented for various stages of those sequential events, as a function of $\mathrm{PAH}$ size. The material presented applied to large molecules in general and was not restricted to the PAH case. The models sought to investigate the radiative, and nonradiative intramolecular processes, including dissociation, that are competitive for conversion of the absorbed ultraviolet photon energy. The essential differences between the Barker and d'Hendecourt approaches were brought out by estimations of the range of physical parameters which delimited the approximate validity of a thermal treatment using Boltzmann statistics or the Planck function as compared with a more formally correct quantum statistical (i. e. non-thermal) approach to energy conversion and dissipation. It was concluded that the thermal approximation was reasonably satisfactory at high (absorbed) photon energies in explaining conversion to an infrared emission situation, but was unsatisfactory at low energies, when the (vibrational) excitation of the excited molecule was of the same order of magnitude as the emitted infrared photons. It was also not valid for discussions of the competitive unimolecular dissociation of excited PAHs, such as dehydrogenation and fragmentation. In those cases the quantum statistical approach was required.

Among points or questions that were brought up in the discussion were the following:

1. A clarification was requested concerning the classical and exact thermal energy curves as a function of temperature presented by John Barker for a hypothetical $C_{50} H_{15}$ molecule. The difference between them reflected the role of very low frequency modes which had relatively high occupation numbers even at low temperatures. 
2. A request for information on experimental evidence in favor of the appropriateness of the models to treat the emission. This led to a discussion on the calculation of density of states. Barker affirmed that exact densities of states were used for species containing up to $20 C$ atoms. This was possible because all vibrational frequencies were known for species in this size range. Vibrational assignments have been made, in particular by Cyvin, for several hydrocarbons up to about $C_{48}$. Since there were great regularities in the way those hydrocarbons behaved, predictions of densities of states could be made based on simple empirical rules. This also made it possible to calculate specific heats. In any case, it appeared that the calculated average thermal energies based on those predictions of densities of states were within a few percent of the exact thermal energies.

The request for experimental information was answered by saying that what one knows actually or potentially, is the physics of isolated molecules. Emphasis was put on the importance of understanding how various properties evolved in going from a single small molecule to larger and larger species until one reached the macroscopic particle domain.

3. The possibility of excitation of collective (electron) modes in PAHs with large numbers of $\pi$ electrons was raised but was relegated to the domain of solid state physics.

4. The minimum number of atoms in a surviving $\mathrm{PAH}$ was discussed variously as 20 and as 60-70. The difference came, in part, from the theoretical treatment i. e., a thermal or a non-thermal approach. The two approaches could also be distinguished by canonical and microcanonical treatments.

5. The effective temperature was different for various pairs of the infrared emission features in d'Hendecourt's presentation. This was perhaps a manifestation of a breakdown of the thermal approximation. It was stressed that the number of carbon atoms, $N_{c}$, derived from the model depended on those ratios and therefore on the infrared transition probabilities. Improved information on the latter should lead to better estimates of $N_{c}$. Recent results on infrared absorption spectra of PAHs clustered in $C s I$ pellets and isololated in matrices were discussed. The importance of obtaining data on isolated molecules was stressed in view of new matrix results.

6. The significance and validity of basing calculations of temperature etc. on the intensity ratio of the $11.3 / 3.3 \mu \mathrm{m}$ bands was discussed. It was queried as to whether larger species could be contributing to the $11.3 \mu \mathrm{m}$ emission, but not the $3.3 \mu \mathrm{m}$ emission. The ratio of those two lines cannot represent the complete distribution of molecules. The concept of temperature was reduced to that of a color temperature that basically represented a system with some sort of average molecular size, and average temperature. It would be useful to better relate the different infrared bands to different average size molecules, bigger ones at larger, smaller at shorter wavelengths.

7. Questions were raised about the statistical distribution function and its relation to Boltzmann distributions. It was pointed out that after internal conversion of the electronic excitation into vibrational excitation took place, the vibrational state population reflected the ratio of the density of states in which the particular vibrational mode was excited to the total densities of states accessible to the excited molecule and so does not necessarily give a 
Boltzmann distribution. This created some confusion as to the use of the concept of temperature under these circumstances. The Boltzmann distribution implied a thermal approach; this type of distribution was not necessary under exact counts of the density of states (i.e., the non-thermal model).

8. The approximations inherent in an assumed one-photon absorption of 9-10 eV energy were discussed, when it was pointed out that the irradiating star would emit photons that were usefully absorbed by the PAHs over the range 3-10 eV. The fact that the electronic absorption of a PAH consisted of relatively narrow bands must be taken into account in calculating the energy distribution. Furthermore, if there was a size-range of molecules, this would create sets of absorption regions. It was thought that perhaps a convolution of a Planck function and a particular form of absorption cross-section might mimic a thermal distribution. The appropriate calculation has not been carried out.

9. It. was pointed out that dust particles in a very hot plasma $\left(\sim 10^{7} \mathrm{~K}\right)$ also had an excess of $12 \mu \mathrm{m}$ and $25 \mu \mathrm{m}$ emission, related in part to the stochastic nature of heating of dust by electron collisions. That environment would give rise to particular distributions of grain size. What were the smallest surviving grains in such an astrophysical plasma? It was replied that a size estimation could always be made if there were pairs of line emissions. If a species less than 5 atoms resulted it was doubtful whether either model would be valid.

10. The method of determining the polarization of emitted radiation was commented on and the different factors involved were discussed. Different polarization could be expected from different bands according to the direction of the transition moment. Observations of UIR bands by Léger et al. did not show polarization to within $1 \%$. Improved modelling gave results consistent with this observation. 